University of Wollongong

Research Online

Faculty of Informatics - Papers (Archive)

Faculty of Engineering and Information

Sciences

28-10-2007

\title{
Realising WACNet Through a Zigbee-based Architecture
}

\author{
A. Desmet \\ University of Wollongong \\ F. Naghdy \\ University of Wollongong, fazel@uow.edu.au \\ Monserrat Ros \\ University of Wollongong, montse@uow.edu.au
}

Follow this and additional works at: https://ro.uow.edu.au/infopapers

Part of the Physical Sciences and Mathematics Commons

\section{Recommended Citation}

Desmet, A.; Naghdy, F.; and Ros, Monserrat: Realising WACNet Through a Zigbee-based Architecture 2007. https://ro.uow.edu.au/infopapers/649 


\title{
Realising WACNet Through a Zigbee-based Architecture
}

\author{
Abstract \\ The development of a novel architecture called Wireless Ad-hoc Control Networks (WACNets) is reported. \\ WACNet (Wireless Ad-hoc Control Network) is a novel concept developed by Intelligent Mechatronics \\ Research Centre, University of Wollongong, towards providing a framework for highly distributed \\ intelligent wireless control networks. A WACNet consists of intelligent nodes based on IEEE 1451 Smart \\ Sensor with a wireless means of communication (BlueTooth). The focus in this paper is on designing a \\ suitable wireless communication system for this network. In the earlier design. the wireless \\ communications was developed based on Bluetooth and WiFi standards. While being successful, they \\ showed some limitations for control networks applications. In the latest test-bed, ZigBee RF standard is \\ deployed. This has opened new perspectives for wireless control networks. ZigBee modules can form a \\ flexible ad-hoc network with no infrastructure. ZigBee is also selfconfiguring, offers interesting \\ transmission ranges and is the most energy efficient compared to the previous options. The WACNet \\ architecture is introduced. Different test-beds developed are described. The performance of the ZigBee \\ based system is compared with the previous implementations in the context of latency and throughput. \\ Results are presented, and some conclusions are drawn.| \\ Disciplines \\ Physical Sciences and Mathematics

\section{Publication Details} \\ This conference paper was originally published as Desmet, A, Naghdy, F, Ros, M, Realising WACNet \\ Through a Zigbee-based Architecture, IEEE Sensors 2007, 28-31 Oct, 143-146.
}




\title{
Realising WACNet Through a Zigbee-based Architecture
}

\author{
Antoine Desmet, Fazel Naghdy, Montserrat Ros \\ School of Electrical, Computer and Telecommunication Engineering, \\ University of Wollongong, Wollongong, Australia, 2522 \\ Email:ad011@uow.edu.au
}

\begin{abstract}
The development of a novel architecture called Wireless Ad-hoc Control Networks (WACNets) is reported. WACNet (Wireless Ad-hoc Control Network) is a novel concept developed by Intelligent Mechatronics Research Centre, University of Wollongong, towards providing a framework for highly distributed intelligent wireless control networks. A WACNet consists of intelligent nodes based on IEEE 1451 Smart Sensor with a wireless means of communication (BlueTooth). The focus in this paper is on designing a suitable wireless communication system for this network. In the earlier design. the wireless communications was developed based on Bluetooth and WiFi standards. While being successful, they showed some limitations for control networks applications. In the latest test-bed, ZigBee RF standard is deployed. This has opened new perspectives for wireless control networks. ZigBee modules can form a flexible ad-hoc network with no infrastructure. ZigBee is also selfconfiguring, offers interesting transmission ranges and is the most energy efficient compared to the previous options. The WACNet architecture is introduced. Different test-beds developed are described. The performance of the ZigBee based system is compared with the previous implementations in the context of latency and throughput. Results are presented, and some conclusions are drawn.|
\end{abstract}

\section{INTRODUCTION}

The advances made in computer networking have had significant impact on industrial controllers. These control systems were traditionally central and hierarchical where large centrally located mini-computers were connected directly to dumb $\mathrm{I} / \mathrm{O}$ racks through the $\mathrm{I} / \mathrm{O}$ ports of the computer. The first major change took place when a distributed architecture was adopted. In this configuration, a central computer supervised distributed direct digital controllers (DDC) with some local intelligence in the $\mathrm{I} / \mathrm{O}$ modules. In the 1980's, network connectivity was introduced between the supervisory computer system and DDC through dial up link. The supervisory computer ran the proprietary vendor software. The dial up connection was replaced by Ethernet in the early 90's and various control modules in the DDC were linked up by vendor propriety networks. Around 1995, the proprietary software running on the supervisory control was replaced by browser-based front end and more standard network tools such as LONWorks ${ }^{1}$ and BACnet ${ }^{2}$ providing connectivity among the DDCs.

Recent advances in mobile computing, wireless communications, MEMS-based sensor technology, lowpowered analogue and digital electronics, and low-power RF design have created opportunities for the introduction of WACNets (Wireless Ad-hoc Control Networks). This architecture represents a major evolution of traditional wired control networks, as it adds flexibility, promotes local intelligence, for a better control of any process, at lower cost. The concept introduces totally autonomous nodes which, once powered, are able to organize themselves in clusters, and create an organized network. This highly-flexible, infrastructure-less intelligent network concept requires no configuration or intervention. Hence, the system does not require any addressing scheme or any knowledge of the network in use.

In the course of this paper, the WACNet concept will be presented. Some rationale for selection of ZigBee standard for this implementation against Bluetooth will be provided. The experimental set up developed to evaluate the performance of the ZigBee based WACNet in the context of throughput and latency is described. The results of the experiments are reported and compared with the previous implementations. Finally, some conclusions are drawn.

\section{WACNETS}

The concept of Wireless Ad-hoc Control Networks (WACNet) represents a new stage in the evolution of distributed control and monitoring. It explores a framework for organic, evolutionary and scalable integration a large number of nodes with sensing and/or actuation, local intelligence and control, data processing and communication capabilities.

A WACNet consists of a set of geographically distributed intelligent and heterogeneous nodes. Each node consists of a processing unit, wireless communication unit, and transducer

\footnotetext{
${ }^{1}$ Full details are provided at http://www.echelon.com/.

${ }^{2}$ Full details are provided at http://www.bacnet.org/.
} 
ports which can connect to one or more sensors or/and actuators. The processing unit can perform signal processing and control depending on the services required from the node and the type and the number of sensors/actuators attached to it.

An architecture based on IEEE $1451^{3}$ smart sensor standards are deployed for realization of WACNet. The IEEE 1451 compliant smart sensor is modified to include a short-range wireless communication link as shown in Figure 1. Such system will have the ability to provide selfidentification, self-testing and adaptive calibration.

The previous stage of this project $[1,2]$ was developed based on Bluetooth RF standard. Overall, the Bluetoothbased WACNets proved to be a feasible solution, though there were a number of issues requiring further attention and development. For instance, the Bluetooth standard did not offer the possibility of any ad-hoc routing, and also limited the number of nodes per cluster to seven. It was also not possible to reach battery-powered autonomies beyond a week. The communication range was limited, which meant that an efficient network required to be very dense.

The ZigBee standard, on the other hand, provides adequate solutions to the encountered problems. This is a high-level protocol relying on the IEEE 802.15.4 standard. The IEEE 802.15.4 only defines MAC and PHY layers, offering services similar to Bluetooth, but orientated towards geographically wider networks, lower energy consumption, and slower data rates. The ZigBee standard, on the other hand, starts at the network management layer, and is located above the IEEE 802.15.4 stack. The ZigBee standard can efficiently manage a network of IEEE 802.15.4-compliant nodes. It can be deployed to implement an ad-hoc protocol on ZigBee Routers, and a simple master/slave star topology for clustered End-Devices running on limited power. Practically, the transition to a ZigBee network has made the self-configuration of an ad-hoc network of WACNet nodes feasible, while it has supported the transparent and multi-hop delivery of messages across the entire network. Moreover, the ZigBee modules consume less energy and have a better communication range than Bluetooth modules.

A WACNet is not only a platform for distributed control and sensing, but can execute distributed tasks. Based on the observation that any node in a control network requires a microcontroller to manage the communications, this concept aims at illustrating the possibility of utilising the free time of each local microcontroller to perform a portion of the global task. This can include operations such as driving the sensors and actuators, performing data conditioning and carrying out signal processing.

\section{TEST-BED AND EXPERIMENTS}

A test-bed consisting of nine nodes is developed. Each node is composed of a microcontroller (ATMEL AVR 8-bit microcontroller: MEGA 32 on development boards) and a ZigBee-compatible transceiver: MaxStream's Xbee chips. The node runs a single program which handles the control task and the networking task at the same time, but the module driver is run in the background. The low-level module driver is similar to the one found in any router. It uses circular FIFO buffers and always gives priority to receiving data. When the node is not busy receiving or sending, and not busy with the control task, it reads and processes the information received from the XBee chip.

The network is enhanced by a Network CAPable (NCAP) node. This allows bidirectional communication between any node of the network and a control station

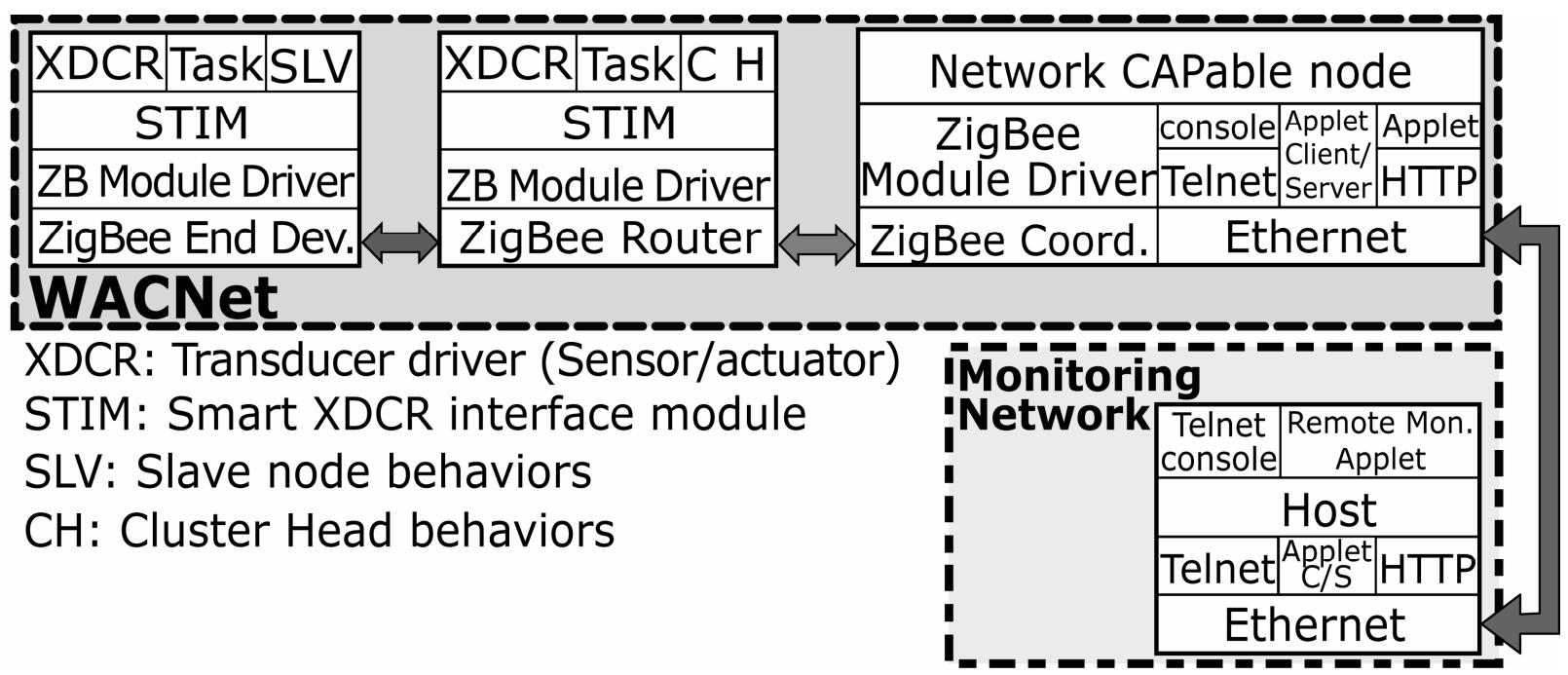

Fig. 1. WACNet nodes and network structure

\footnotetext{
${ }^{3}$ IEEE P1451, Draft Standard for a Smart Transducer Interface for Sensors and Actuators, http://ieee1451.nist.gov/intro.htm.
} 
connected to an Ethernet LAN. The NCap node converts the HTTP data into direct orders, sent via the ZigBee module. Typically, this allow the whole system to be visualized and monitored using just a web browser running a Java applet.

All the nodes within the network run a self-organization algorithm that will determine which ones are meant to become cluster heads (and therefore become ZigBee Routers). The creation of clusters simplifies the network communications and reduces the complexity of the routing task. It also eases the control of the process: the nodes that work towards a common goal are preferably part of the same cluster, and are able to communicate directly.

The first stage of study, described in this paper, focused on verifying the capability of the microcontroller to handle both the control and communications processes including creating, receiving and transmitting messages to other nodes.

In the first experiment, the processing, transmission and receiving times are measured. This is achieved by monitoring the state of an output pin with an oscilloscope. In the program, this pin is set to high at the beginning of an operation, and at low when it is complete. The outcome will produce numerical values of the latencies.

In the second experiment, two ZigBee routers exchange data as fast as possible. In order to get the most accurate results, the buffers are sized to fit only one frame. The microcontroller must first generate a frame that can be read by the module, (including headers and escape characters handling). The frame is then written to the transmission buffer, so that the background task can transmit it to the ZigBee module, via the USART (9,600 bauds). An acknowledgement is sent back and must also be read. For each configuration, the experiment begins with a working configuration, and then the delay between the transmissions of consecutive messages is progressively decreased, until the buffer overflows. The last correct value is the maximum throughput of the node.

\section{EXPERIMENTAL RESULTS AND ANALYSIS}

The outcomes of the first test are illustrated in Figure 2.

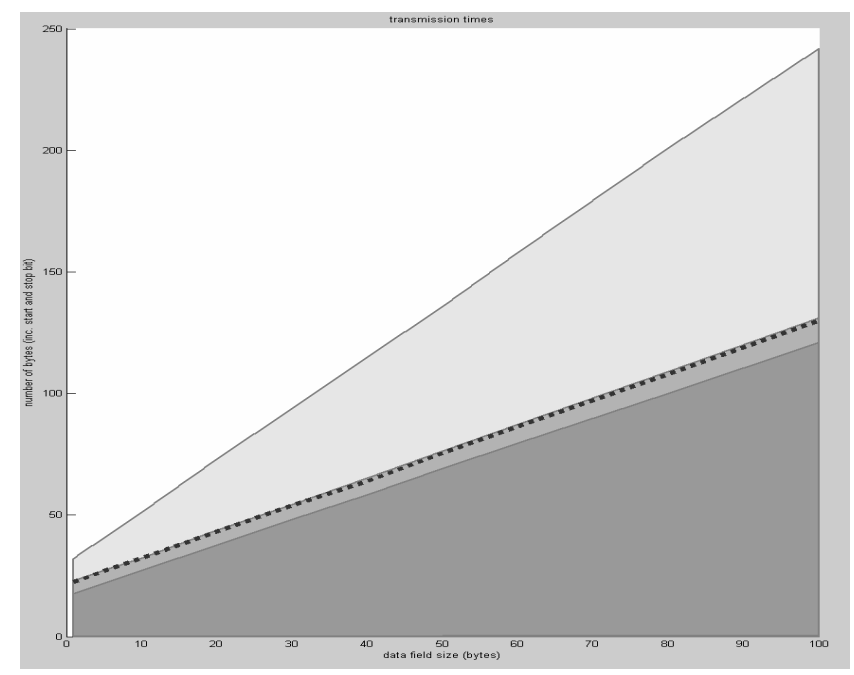

Fig. 2. Exp. 1: transmission times versus payload size
This diagram shows the variation of time ( $\mathrm{Y}$-axis, from 0 to $250 \mathrm{~ms}$ ) versus payload size (X-axis, from 1 to 100 bytes). Each area corresponds to a step of the transmission process. They represent transmission to the ZigBee module (source node), ZigBee wireless transmission, and reception from the ZigBee module (destination node), from the darkest to the lightest region respectively. The initial computation time is also plotted, but is too small to be visible. The dashed line corresponds to the instant at which the acknowledgement is sent.

As expected, the transmission times are linear. The transmission time of a message cannot be below $30 \mathrm{~ms}$, because the smallest frame still has addressing and checksum overhead, for both transmission and reception. The computation time is insignificant. The message transmission time is also very small. In fact, the delay is mainly the data transfer times from the microcontroller to the ZigBee module. This transfer time can be expressed by the following equation:

$$
t_{n_{\text {bytes }}}=\left(H_{\text {bytes }}+p_{\text {bytes }}\right) \times T_{k}
$$

Where $H_{\text {bytes }}$ is the header size (constant), $p_{\text {bytes }}$ is the size of the payload (variable), and $T_{k}$ is the speed of transmission in s/byte, (depends mostly on the USART speed).

In conclusion, the transfer time of a given amount of data is a linear function with offset, increasing with the size of the payload. The larger the payload, the higher will be the latency. From this strict point of view, the best solution to achieve low latency appears to be sending frames with a small payload. Small frames containing just a few sensory values can achieve latencies below $40 \mathrm{~ms}$.

The results of the second experiment are shown in figure 3. The $X$-axis is the payload size from 1 to 100 bytes, and the $\mathrm{Y}$-axis is the byterate, from 0 to 900 bytes of information per second.

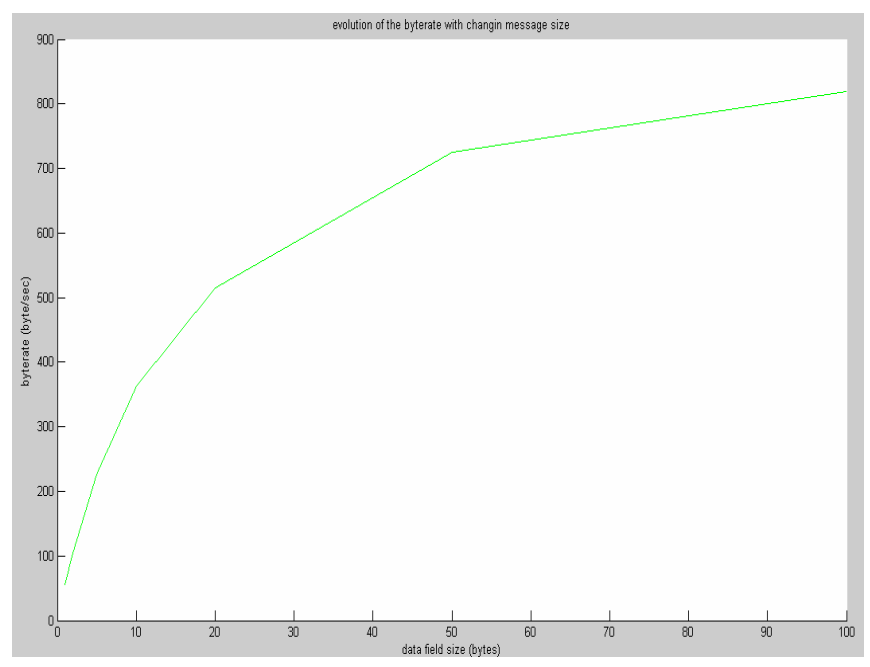

Fig. 3. Exp. 2: byte rate versus payload size 
In this experiment, the maximal transmission speed has been acquired with payload size as a parameter. This graph clearly demonstrates that the best throughput is achieved when the payload is maximum. A maximum rate of 800 bytes per seconds is reached when the payload size is the highest possible magnitude. This diagram can be approximated by :

$$
\frac{b_{\text {bytes }}}{t_{\text {sec. }}}=\frac{p_{\text {bytes }}}{\left(H_{\text {bytes }}+p_{\text {bytes }}\right)} \times T_{k}
$$

This implies that the byterate $(b / t)$ depends directly on the payload over frame size ratio: $p /(H+p)$. In order to reach the best throughput, the payload should be as large as possible, so that the ratio is maximum. This is due to the fact that, while the baudrate of the USART remains the same, the amount of byte required to transmit the information increases when the payload size decreases:

$$
T x_{\text {bytes }}=n_{\text {bytes }}+\frac{H_{\text {bytes }} \cdot n_{\text {bytes }}}{p_{\text {bytes }}}
$$

Where $T x_{\text {bytes }}$ is the total number of bytes that are sent through the USART during the transmission of $n_{\text {byte }}$ bytes of data distributed over a number of frames, each containing $p_{\text {bytes }}$ of payload. This equation is illustrated in figure 4 , for a datalength of 1,000 bytes.

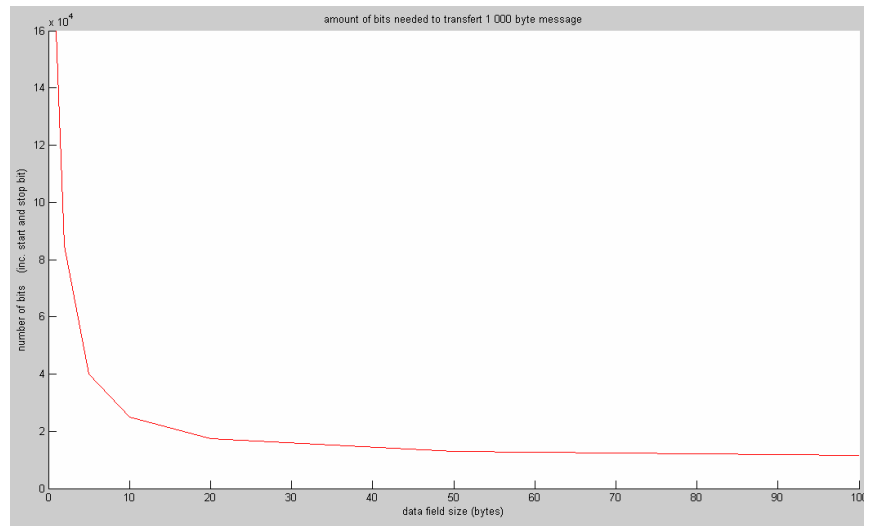

Fig. 4. Exp. 2: Total number of bytes transmitted in order to send $1,000 \mathrm{~b}$. of information, depending on the payload size

Figure 4 illustrates the payload over frame size ratio mentioned above. It represents the amount of bytes that must be transmitted in order to send 1000 bytes of data (parameter $n_{\text {byte }}$ in eqn. 3$)$. The $\mathrm{X}$-axis represents the payload size ( $p_{\text {bytes }}$ in eqn. 3) from 1 to 100 bytes, and the $\mathrm{Y}$-axis is the amount of transmitted bytes ( $T x_{\text {bytes }}$ in eqn. 3 ) from 0 to 160,000 . The plot has a $y=1 / x$ shape, that matches equation 3 . It reveals the fact that when the payload size is reduced, the information must be broken down into more frames. Thus, the amount of frames transmitted increases and so does the overhead. When the payload on frame size ratio decreases, there is more overhead to transmit, therefore less information can be transferred in a given time, which explains the decreasing byterate.

From the results of these two experiments, it can be clearly concluded that a choice must be made between latency and throughput.

\section{CONCLUSION}

In the course of the paper, the concept of WACNet has been introduced and its characteristics have been described. The ZigBee standard, the communication media replacing the Bluetooth, along with its advantages over the Bluetooth standard have been explained. The platform designed to efficiently deploy the ZigBee module is presented. Finally, some benchmark tests have been conducted to obtain transfer speeds, latency and throughput of the proposed system.

The study has shown that the ZigBee standard is a better option for deployment in WACNets than Bluetooth, from many points of view.

The experiments have also proved that it is not possible to achieve small latencies and high throughputs at the same time. The average payload size must be optimized to maintain an acceptable level of latency while not losing too much time, resources and energy during the transmission and computation of almost-empty frames.

In the next stage of the project, the latencies will be significantly reduced by accelerating the USART speed, and ultimately by processing all of the ZigBee network management and communications task in the node's microcontroller itself.

\section{REFERENCES}

[1] S. Bu, F. Naghdy, "Wireless ad-hoc control networks", 3rd IEEE International Conference on Industrial Informatics (INDIN '05). (2005) pp 839- 844

[2] S. Bu, F. Naghdy, "Service Discovery in Wireless Ad-hoc Control Networks", Proceedings of the 2005 International Conference on Intelligent Sensors, Sensor Networks and Information Processing Conference, 2005. pp: 157- 162 